\title{
BEAM MODE EXPANSION OF CORRUGATED CONICAL HORNS WITH PHASE CORRECTING LENS: APPLICATION TO RADIOASTRONOMY RECEIVERS
}

\author{
E. GARCÍA ${ }^{1, *}$, L. DE HARO ${ }^{1}$, C. O’SULLIVAN ${ }^{2}$, G. CAHILL ${ }^{2}$, \\ J. A. LÓPEZ FERNÁNDEZ ${ }^{3}$, F. TERCERO ${ }^{3}$, B. GALOCHA ${ }^{1}$ and J. L. BESADA ${ }^{1}$ \\ ${ }^{1}$ Grupo de Radiación, Dept. S.S.R., Universidad Politécnica de Madrid, Ciudad Universitaria, \\ 28040 Madrid, Spain; ${ }^{2}$ Department of Physics, National University of Ireland, Maynooth, \\ Maynooth, Co. Kildare, Ireland; ${ }^{3}$ Centro Astronómico de Yebes, Instituto Geográfico Nacional, \\ Yebes, Guadalajara, Spain \\ ( ${ }^{*}$ author for correspondence, e-mail: legarcía@tsc.uc3m.es)
}

(Received 14 January 2004; accepted 26 April 2004)

\begin{abstract}
A classical radioastronomy receiver is fed with a corrugated horn and an independent lens, both placed in a cryostat to lower the noise temperature. The beam is focused and directed using a combination of elliptical and plane mirrors. This paper proposes modifying the initial feeding system by placing the lens onto the horn aperture, thereby allowing a size reduction of the horn and lens, and a simplification of their mechanical design. The profiled lens is shaped to correct the phase error on the horn aperture. A quasi-optical model of the horn-plus-lens system has been developed using a Beam Mode Expansion (BME). Results using both a hyperbolic-planar lens and a spherical-elliptical lens, as well as results obtained by using Geometrical Optics (GO) with a Kirchoff-Huygens integration to get the far-field pattern, have been compared with measurements. As a direct application, a full focusing system for the new 40-m radiotelescope at the "Centro Astronómico de Yebes" is presented for the 22, 30 and $45 \mathrm{GHz}$ bands. This paper has developed a QO model for a corrugated conical horn with a phase-correcting lens.
\end{abstract}

Key words: beam mode expansion, quasi-optical systems, radioastronomy, radiotelescope

\section{Introduction}

Radioastronomy receivers have feeder systems formed by corrugated conical horns, a focused lens, an elliptical mirror and a set of several plane mirrors (Chu, 1983; Tuovinen et al., 1992). The first two elements are placed in a cryostat to lower the system noise temperature (Goldsmith, 1997). Quasi-Optical Theory (QO) (Goldsmith, 1997; García et al., 2002) covers the analysis of this structure (called a Gaussian telescope), while a Beam Mode Expansion (BME) optimizing fundamental-mode power (Wylde, 1984) is applied to the corrugated conical horn. Applying both theories simultaneously works properly provided that the lens is many horn confocal distances away (Goldsmith, 1997). However, if the lens is placed in the near field of the horn, the field radiated by the lens is less described (Tuovinen et al., 1992). This is the case when a lens profile is chosen to correct a horn phase error. Clarricoats and Saha (1969) and Kildal et al. (1984) have worked

Experimental Astronomy 15: 171-193, 2003.

(c) 2003 Kluwer Academic Publishers. Printed in the Netherlands. 
with such a systems with excellent results. In this paper we expand that analysis from the point of view of the QO theory.

Figure 1 shows a comparison between GO (with a Kirchoff-Huygens integration in the aperture plane to get the far-field pattern) and QO for a horn with a meniscus lens at $30 \mathrm{GHz}$. A BME is used to model the corrugated horn (Wylde, 1984; García et al., 2003) and the effect of the lens is added via an ABCD matrix. The horn axial length is $105.3 \mathrm{~mm}$ and horn aperture radius is $49.1 \mathrm{~mm}$. The refractive index of lens material is 1.45 . As can be seen, the radiated pattern is not accurately predicted by the QO. This paper proposes the use of a more accurate procedure where the $\mathrm{BME}$ is applied to the pattern produced by the horn-plus-lens system.

The structure of this paper is as follows. In Section 2, a model of a horn with a spherical-elliptical lens is developed along with the BME method and its optimization procedure. A BME is used to model a horn plus hyperbolical-planar lens system in Section 3. In Section 4, we validate the BME proposed for the hyperbolical-planar lens in Section 3 by means of a system working at $7.25 \mathrm{GHz}$. In Section 5, we consider the spherical-elliptical lens and validate the method by constructing a radioastronomical receiver at $30 \mathrm{GHz}$. In this section we also present an optical solution for other bands of the new 40-m radiotelescope of the "Centro Astronómico de Yebes". Finally, in Section 6 we discuss the results and consider their implications for the $22 \mathrm{GHz}$ channel of the new radiotelescope.

In radioastronomy there are several scientific observation bands of interest, from $2 \mathrm{GHz}$ up to higher than $300 \mathrm{GHz}$ (Chu, 1983; Tuovinen et al., 1992). An individualized study of the problem is necessary for each observation band (Chu, 1983; Goldsmith, 1997), in order to design the feeds and the focalization elements such as lenses and conical or plane mirrors (Chu, 1983; Tuovinen et al., 1992; Goldsmith, 1997; García et al., 2002; Wylde, 1984). This paper is related with the necessity of the focalization of the new 40-m Nasmyth-Cassegrain radiotelescope of the Centro Astronómico de Yebes in Spain with F/D ratio of 7.9 (García et al., 2003).

Our aim is to cover several bands from $120 \mathrm{GHz}$ to $2 \mathrm{GHz}$. There is a dimension constraint in the receiver cabin for the situation of all the receivers, so it is suitable to share the maximum number of focalization elements for each observation band. In Figure 2 the receiver cabin and the physical dimension available for the situation of the receivers and their focalization elements are represented (García et al., 2003). It can be seen (Figure 1) that the cabin presents two Nasmyth asymmetric sections centered on M4 and M4' mirrors optical axis that can be selected by means of Nasmyth mirror M3.

To deal with the focalization problem of the different radiotelescope bands, the quasi-optics theory (QO) is used in this paper (Chu, 1983; Goldsmith, 1997; Wylde, 1984; Clarricoats and Saha, 1969). In order to achieve the proper focalization of the radiotelescope two fundamental requirements are necessary: the first one is that the subreflector (and so the main reflector) has a suitable taper (illumination level in the edge), of $12 \mathrm{~dB}$ taper for optimum aperture efficiency. The second requirement is the existence of a perfect focalization, in terms of geometry optics 


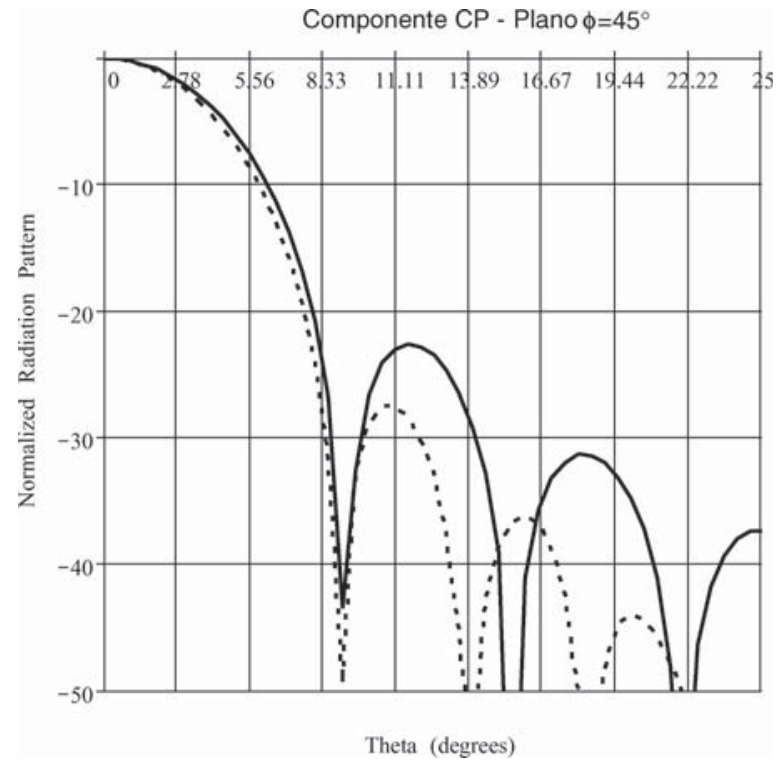

Figure 1. Copolar radiation pattern $\left(\phi=45^{\circ}\right)$ of a corrugated horn and a spherical-elliptical lens: solid GO, dotted QO with 31 modes.

(GO) (Goldsmith, 1997), that feeders are situated in the system foci. In Figure 3 the geometry of the radiotelescope is presented whose fundamental parameters are:

- Primary

- Main reflector diameter $40 \mathrm{~m}$

- Fm/Dm 0.375

- Fm $15 \mathrm{~m}$

- $\Phi \mathrm{V} \quad 67.38^{\circ}$

- hp $6.667 \mathrm{~m}$

- Vertex hole $3.170 \mathrm{~m}$

- Cassegrain configuration

- FED 7.909

- Fe $316379 \mathrm{~m}$

- $\operatorname{\Phi r} 3.621^{\circ}$

- Magnification, M 21.0919

- Lv $1.204 \mathrm{~m}$

- Fc $26600 \mathrm{~m}$

- Lr 25396 m

- $g \quad 11.6 \mathrm{~m}$ 


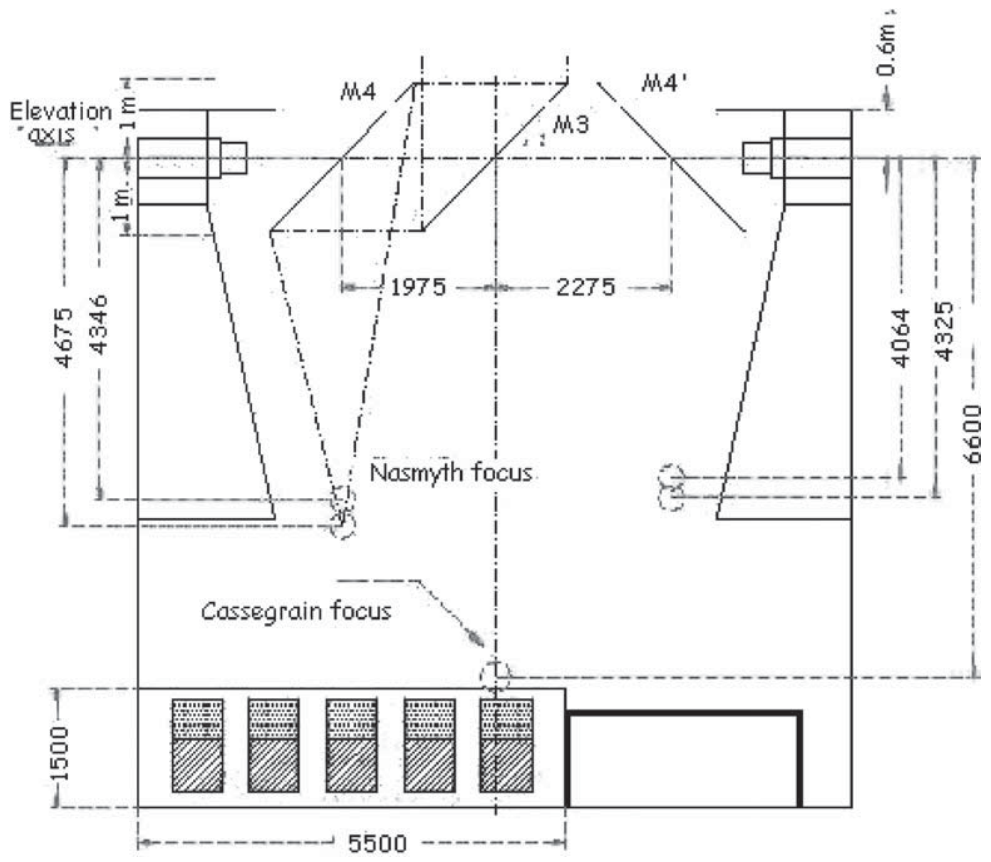

Figure 2. Radiotelescope receiver cabin.

- gl $5.0 \mathrm{~m}$

- hs $7.129 \mathrm{~m}$

- Subreflector geometry

- Ds $3.28 \mathrm{~m}$

- ex 1.099555

- prof $0.5207 \mathrm{~m}$

- Dvs $0.2635 \mathrm{~m}$

\section{Horn with spherical-elliptical lens}

Consider a corrugated conical horn placed along $z$ axis and propagating an hybrid mode HE11 (linearly polarized- $\hat{x}$ ), the aperture field can be described as (Clarricoats and Olver, 1984):

$$
\begin{aligned}
E_{x}(\theta, \phi) & =F_{1}(\theta)\left(\cos (\theta) \cos ^{2}(\phi)+\sin ^{2}(\phi)\right) \\
E_{y}(\theta, \phi) & =F_{1}(\theta)(\cos (\theta)-1) \sin (\phi) \cos (\phi)
\end{aligned}
$$




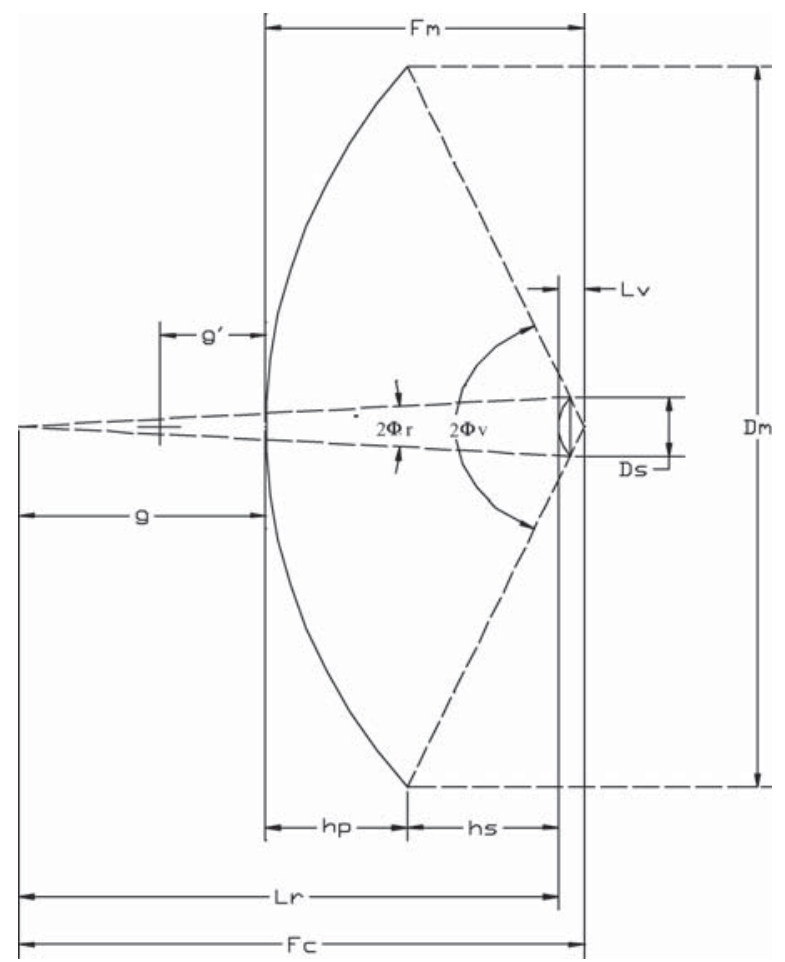

Figure 3. Radiotelescope geometry.

where,

$$
F_{1}(\theta)=\frac{P_{v}^{1}(\cos (\theta))}{\sin (\theta)}+\frac{d\left(P_{v}^{1}(\cos (\theta))\right)}{d \theta}
$$

and $P_{v}^{m}(\cos (\theta))$ is the Legendre polynomial of the first kind of order $m(m=1)$ and degree $v$. As the hybrid mode HE11 is excited, the order $m$ takes the value 1.The degree $v$ is the root of the function $P_{v}^{1}\left(\cos \left(\psi_{\mathrm{e}}\right)\right)$, where $\psi_{\mathrm{e}}$ is the half-flare angle.

A Beam Mode Expansion can be applied to a horn with a phase correcting lens (spherical-elliptical profile). The lens aperture field is calculated from the horn field by applying Jasik's expressions (Jasik, 1961) for the conservation of power in a differential area, giving:

$$
\begin{aligned}
& E_{\text {apx }}(\rho, \phi)=N(\theta(\rho)) F_{1}(\theta(\rho))\left(\cos (\theta(\rho)) \cos ^{2}(\phi)+\sin ^{2}(\phi)\right) \\
& E_{\text {apy }}(\rho, \phi)=N(\theta(\rho)) F_{1}(\theta(\rho))(\cos (\theta(\rho))-1) \sin (\phi) \cos (\phi)
\end{aligned}
$$


where the conservation of power factor is:

$$
N(\theta)=\frac{1}{n-1} \sqrt{\frac{(n-\cos (\theta))^{3}}{n \cos (\theta)-1}}
$$

and

$$
\cos (\theta(\rho))=\frac{\frac{n \rho^{2}}{f^{2}(n-1)^{2}}+\sqrt{1-\frac{\left(n^{2}-1\right) \rho^{2}}{f^{2}(n-1)^{2}}}}{1+\frac{\rho^{2}}{f^{2}(n-1)^{2}}}
$$

$n$ is the refractive index of the lens material and $f$ is the elliptical vertex length (Milligan, 1985).

BME is based on the idea that any aperture function described in cylindrical components can be expressed in terms of generalized Laguerre-Gauss functions:

$$
\begin{aligned}
E_{\mathrm{ap}}(\rho, \phi)= & \sum_{p=0}^{\infty} \sum_{m=0}^{\infty} A_{\mathrm{pmy}}\left(\frac{2 \rho^{2}}{W^{2}}\right)^{m / 2} L_{p}^{m}\left[\frac{2 \rho^{2}}{W^{2}}\right] e^{-\rho^{2} / W^{2}} \sin (m \phi) \cdot \hat{y} \\
& +\sum_{p=0}^{\infty} \sum_{m=0}^{\infty} B_{\mathrm{pmx}}\left(\frac{2 \rho^{2}}{W^{2}}\right)^{m / 2} L_{p}^{m}\left[\frac{2 \rho^{2}}{W^{2}}\right] e^{-\rho^{2} / W^{2}} \cos (m \phi) \cdot \hat{x}
\end{aligned}
$$

with $W$ an arbitrary constant, $p$ and $m$ taking values $0,1,2,3, \ldots$ and $L_{p}^{m}[x]$ the generalized Laguerre polynomial with orthogonality properties,

$$
\begin{aligned}
& \int_{0}^{\infty} L_{P}^{m}\left[\frac{2 \rho^{2}}{W^{2}}\right] L_{Q}^{m}\left[\frac{2 \rho^{2}}{W^{2}}\right]\left(\frac{2 \rho^{2}}{W^{2}}\right)^{m} e^{-2 \rho^{2} / W^{2}} \rho d \rho \\
& =\frac{W^{2}}{4} \frac{\Gamma(m+P+1)}{P !} \cdot \delta_{P Q} .
\end{aligned}
$$

Since the $\hat{x}$ component of the aperture field is simply described by the $m=0$ and $m=2$ terms, and the $\hat{y}$ component by the $m=2$ term, the expansion coefficients can be expressed as:

$$
\begin{aligned}
B_{p 0 x}= & \frac{4}{(W / a)^{2}} \frac{p !}{\Gamma(p+1)} \int_{0}^{1} \frac{1}{2} N(\theta(a u)) F_{1}(\theta(a u))(\cos (\theta(a u))+1) \\
& \times L_{p}^{0}\left[\frac{2 u^{2}}{(W / a)^{2}}\right] e^{-u^{2} /(W / a)^{2}} u d u \\
B_{p 2 x}= & \frac{4}{(W a)^{2}} \frac{p !}{\Gamma(p+3)} \int_{0}^{1} \frac{1}{2} N(\theta(a u)) F_{1}(\theta(a u))(\cos (\theta(a u))-1) \\
& \times\left(\frac{2 u^{2}}{(W / a)^{2}}\right) L_{p}^{2}\left[\frac{2 u^{2}}{(W / a)^{2}}\right] e^{-u^{2} /(W / a)^{2}} u d u
\end{aligned}
$$




$$
\begin{aligned}
A_{p 2 y}= & \frac{4}{(W / a)^{2}} \frac{p !}{\Gamma(p+3)} \int_{0}^{1} \frac{1}{2} N(\theta(a u)) F_{1}(\theta(a u))(\cos (\theta(a u))-1) \\
& \times\left(\frac{2 u^{2}}{(W / a)^{2}}\right) L_{p}^{2}\left[\frac{2 u^{2}}{(W / a)^{2}}\right] e^{-u^{2} /(W / a)^{2}} u d u
\end{aligned}
$$

where the variable $\rho=a u$ is used to normalize the expressions with respect to the aperture radius $a$.

Varying the ratio $W / a$ allows an optimum adjustment of the BME. For instance, a common criterion is the maximization of the fundamental mode power. Wylde (1984) and Murphy (1988) used this for corrugated and smooth conical horns, respectively. Lamb took the complete system into account (Lamb, 1986).

The $\hat{x}$ and $\hat{y}$ components of power that correspond to the $p$ th-mode for $m=0$ or 2 and can be expressed as:

$$
\begin{aligned}
& P_{B_{p 0 x}}(p, W / a) \\
& =\frac{(W / a)^{2} B_{p 0 x}^{2}}{\int_{0}^{1} N(\theta(a u))^{2} F_{1}(\theta(a u)) \cdot\left[(\cos (\theta(a u))+1)^{2}+(\cos (\theta(a u))-1)^{2}\right] u d u} \\
& P_{B_{p 2 x}}(p, W / a) \\
& =\frac{(W / a)^{2} B_{p 2 x}^{2}}{\int_{0}^{1} N(\theta(a u))^{2} F_{1}(\theta(a u)) \cdot\left[(\cos (\theta(a u))+1)^{2}+(\cos (\theta(a u))-1)^{2}\right] u d u} \\
& P_{A_{p 2 y}}(p, W / a) \\
& =\frac{(W / a)^{2} A_{p 2 y}^{2}}{\int_{0}^{1} N(\theta(a u))^{2} F_{1}(\theta(a u)) \cdot\left[(\cos (\theta(a u))+1)^{2}+(\cos (\theta(a u))-1)^{2}\right] u d u}
\end{aligned}
$$

Figures 4 and 5 depict these power expressions (13) and (14) in terms of the ratio $W / a$. Note that Equation (15) is similar to Equation (14). Each mode propagates a maximum power at a specific value of $W / a$. In the case of fundamental mode power optimization, a conjugated gradient optimization gives a value for $W / a$ of 0.712 and $95.3 \%$ of the power is carried by the fundamental mode.

The beam waist appears at the lens aperture where the radius of curvature is infinite. Physically, $W$ is the beam radius in the aperture, as Wylde and Murphy remarked, but in this case, it is also the beam waist. Table I shows the BME coefficients when the power in the fundamental mode is optimized. The mode 


\section{TABLE I}

BME Coefficients with $W / a=0.712$ normalized to $B_{00 x}$

\begin{tabular}{lcr}
\hline Coefficient & $B_{p 0 x}$ & $B_{p 2 x}=A_{p 2 y}$ \\
\hline 0 & 1 & $-7.974 e-3$ \\
1 & $-2.817 e-10$ & $-2.206 e-3$ \\
2 & -0.171 & $4.969 e-6$ \\
3 & -0.095 & $5.662 e-4$ \\
4 & $3.002 e-4$ & $4.807 e-4$ \\
5 & 0.049 & $2.326 e-4$ \\
6 & 0.052 & $2.125 e-5$ \\
7 & 0.030 & $-9.773 e-5$ \\
8 & $3.801 e-3$ & $-1.326 e-4$ \\
9 & -0.016 & $-1.129 e-4$ \\
\hline
\end{tabular}

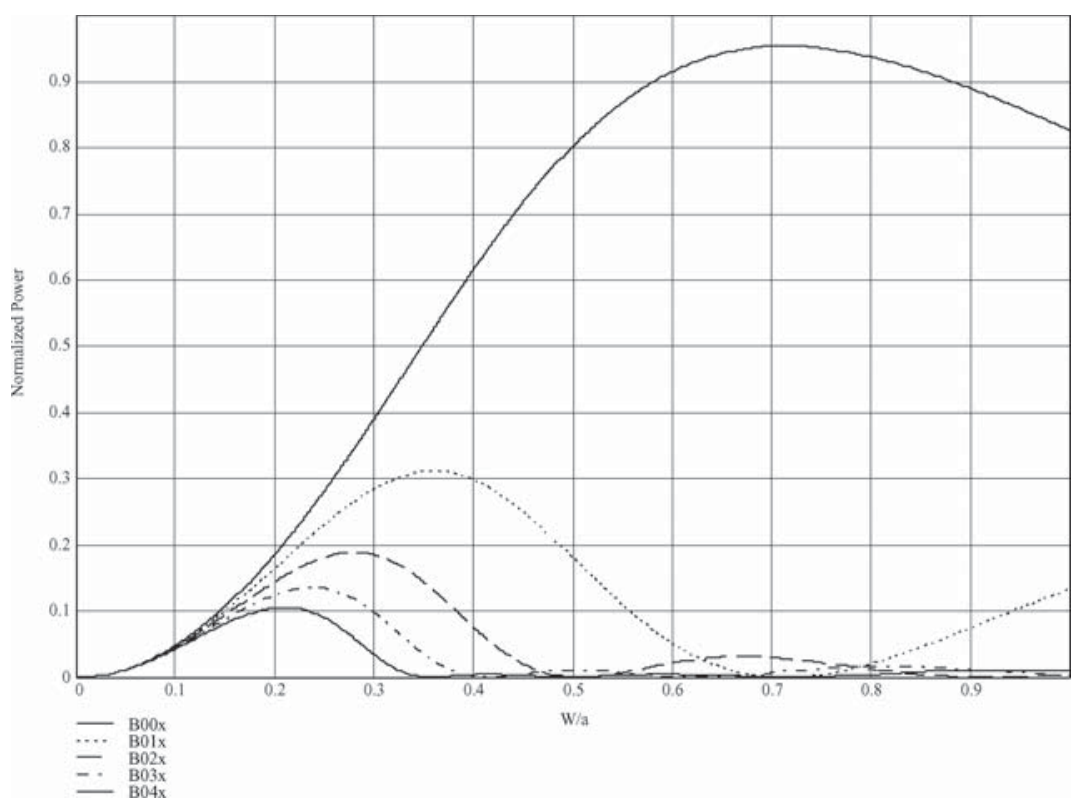

Figure 4. Normalized power vs. $W / a$ for $B_{p 0 x}$ modes for a horn plus spherical-elliptical lens.

coefficients are normalized to the fundamental mode $B_{00 x}$. The difference in scales between the $m=0$ modes and the $m=2$ modes is remarkable. As the normalized power associated with the fundamental mode is high, an analysis based solely on this mode can be conducted. Figure 6 shows the coefficient $B_{p 0 x}$, normalized to $B_{00 x}$, as a function of $W / a$. The $B_{10 x}$ value has been drawn with a thicker 


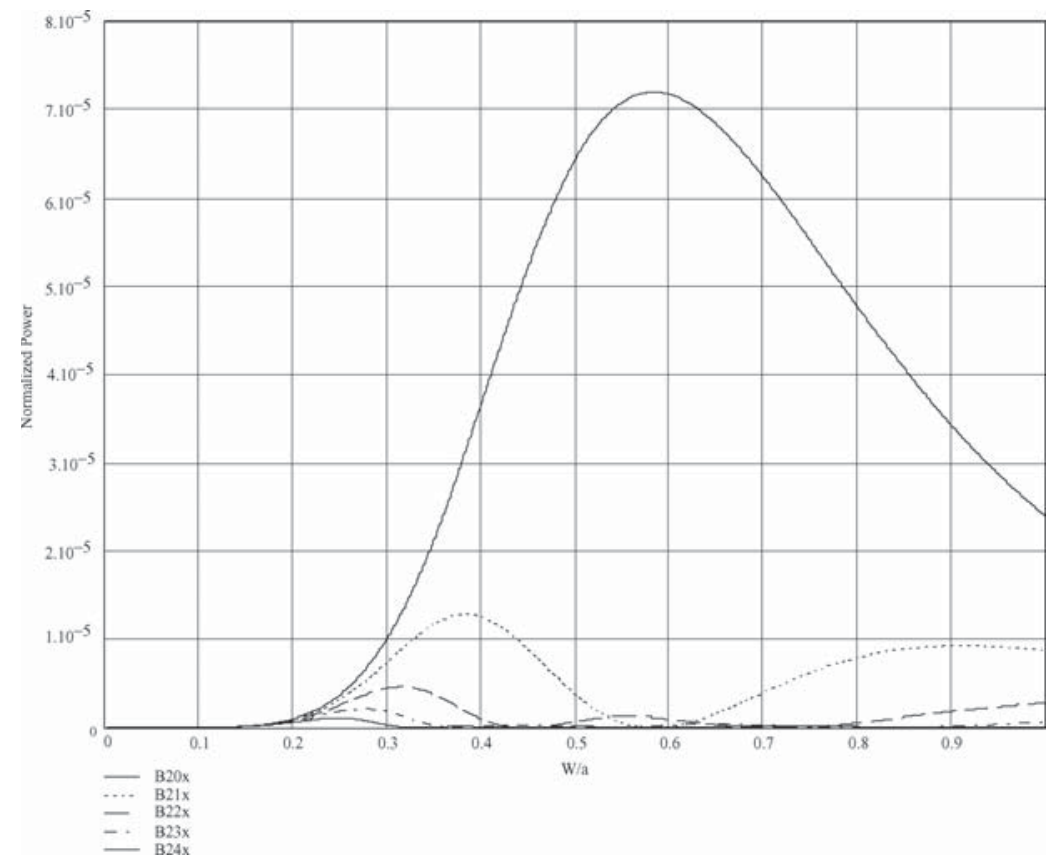

Figure 5. Normalized power vs. $W / a$ for $B_{p 2 x}$ modes for a horn plus spherical-elliptical lens.

line and it can be seen that there is no power associated with it. Minimizing the power in this mode is another method for finding an optimum value for $W / a$ (Lamb, 1986).

\section{Horn with hyperbolic-planar lens}

In case of a hyperbolic-planar lens (Milligan, 1985), the BME analysis is carried out by following the same steps and equations, but changing expression (6) to:

$$
N(\theta)=\sqrt{\frac{(n \cos \theta-1)^{3}}{(n-1)^{2} f^{2} n-\cos \theta}}
$$

and,

$$
\cos (\theta(\rho))=\frac{2 \rho^{2} n \pm \sqrt{4 \rho^{4} n^{2}-4\left(\rho^{2} n^{2}+(n-1)^{2} f^{2}\right) \cdot\left(\rho^{2}-(n-1)^{2} f^{2}\right)}}{2\left(\rho^{2} n^{2}+(n-1)^{2} f^{2}\right)} .
$$

The beam waist is again at the lens output aperture. The fundamental mode power is maximized at $99.83 \%$, when $W / a$ is 0.517 . 


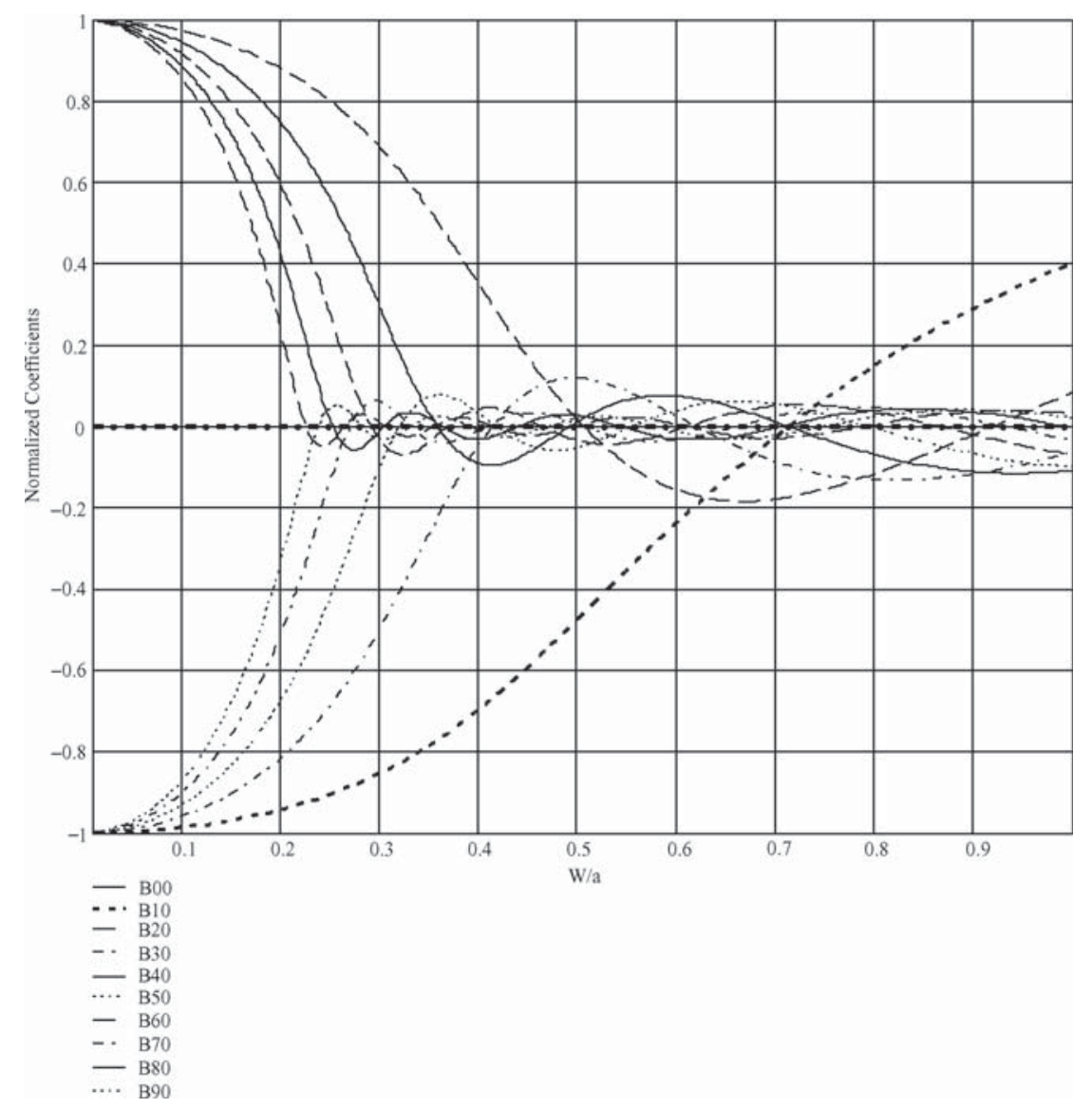

Figure 6. Normalized $B_{p 0 x}$ coefficient in terms of $W / a$ for a horn plus spherical-elliptical lens.

Following the same computation as in the previous section, Figure 7 and 8 represent the normalized power of each mode. Again $A_{p 2 y}$ is similar to $B_{p 2 x}$. Table II shows the values of the coefficients normalized to $B_{00 x}$ for the case of fundamental mode power optimization. As in the previous section, Figure 10 shows the power in each $B_{p 0 x}$ mode of the $W / a$. value. The $B_{10 x}$ value has been represented by a thicker line.

\section{Validation of the hyperbolical-planar lens model}

The BME and GO models were tested by comparing with measurements of a horn plus hyperbolical-planar lens system. 


$$
\pi \cdot \int_{0}^{1}\left[2 \cdot\left(\left|\operatorname{Eap}_{\mathrm{m} 0 \mathrm{x}}(\operatorname{th}(\mathrm{a} \cdot \mathrm{u}))\right|\right)^{2}+\left(\left|\operatorname{Eap}_{\mathrm{m} 2 \mathrm{x}}(\operatorname{th}(\mathrm{a} \cdot \mathrm{u}))\right|\right)^{2}\right] \cdot \mathrm{udu}
$$

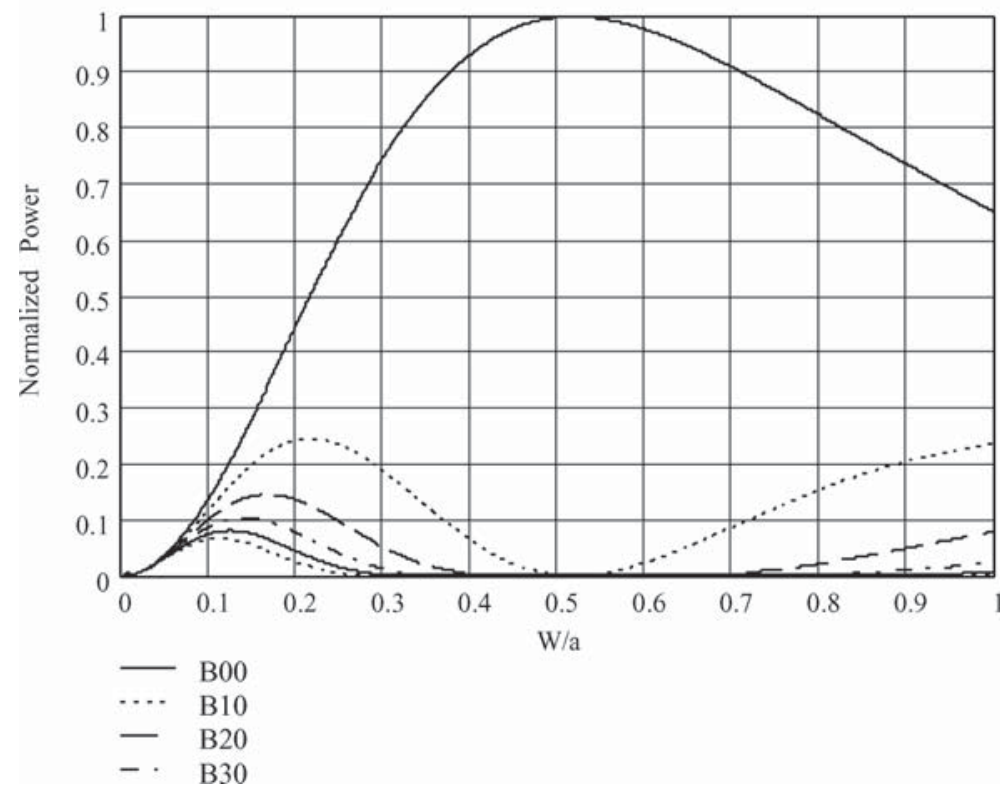

Figure 7. Normalized power in terms of $W / a$ for $B_{p 0 x}$ modes for a horn plus hyperbolical-planar lens.

The output electric field can be expanded as follows:

$$
\begin{aligned}
E_{x}(r, z, \phi)= & e^{\left(\frac{r^{2}}{W^{2}}-\frac{j \pi r^{2}}{\lambda \cdot R(z)}-j k z-j \phi_{0}(z)\right)} \\
& \times\left[\begin{array}{l}
\sum_{m=0}^{M_{\max }} B_{p 0 x} \cdot L_{p}^{0}\left(\frac{2 r^{2}}{W(z)^{2}}\right) e^{-2 j m \phi_{0}(z)}+ \\
\left.\sum_{m=0}^{M_{\max }} B_{p 2 x} \cdot \frac{2 r^{2}}{W(z)^{2}} \cdot L_{p}^{2}\left(\frac{2 r^{2}}{W(z)^{2}}\right) e^{-2 j \cdot(2 m+2) \phi_{0}(z)}\right]
\end{array}\right] \cdot \cos (2 \phi) \\
E_{y}(r, z, \phi)= & e^{\left(\frac{-r^{2}}{W^{2}}-\frac{j \pi r^{2}}{\lambda \cdot R(z)}-j k z-j \phi_{0}(z)\right)} \\
& \cdot \sum_{m=0}^{M_{\max }} B_{p 2 y} \cdot \frac{2 r^{2}}{W(z)^{2}} \cdot L_{p}^{2}\left(\frac{2 r^{2}}{W(z)^{2}}\right) e^{-2 j \cdot(2 m+2) \phi_{0}(z)} \cdot \sin (2 \phi)
\end{aligned}
$$

with $M_{\max }$ the number of modes used in the BME, $R(z)$ the curvature radius, $W(z)$ the beam radius and $\phi_{0}(z)$ the initial phase slippage (Goldsmith, 1997). 


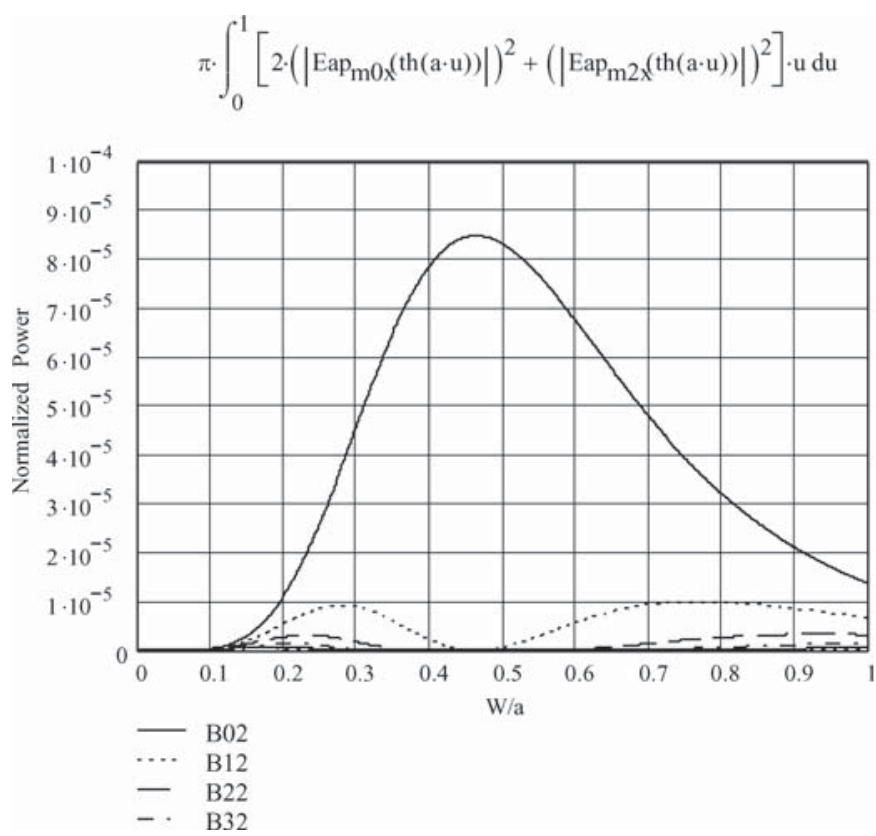

Figure 8. Normalized power in terms of $W / a$ for $B_{p 2 x}$ modes for a horn plus hyperbolical-planar lens.

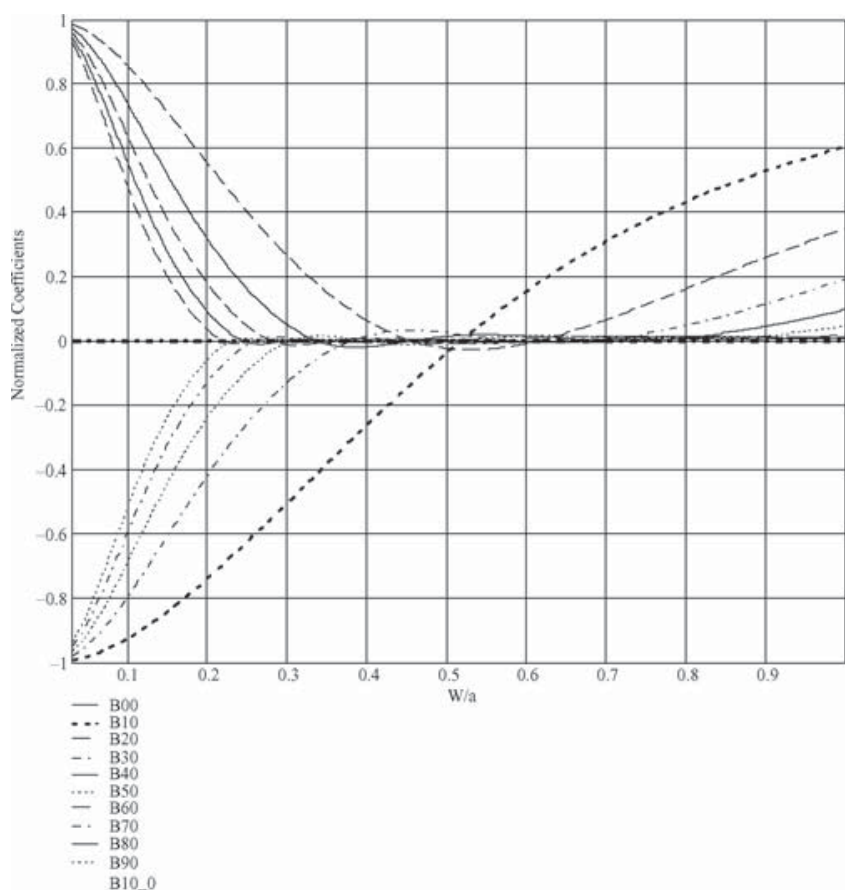

Figure 9. Normalized $B_{p 0 x}$ coefficient as a function of $W / a$ for a horn plus hyperbolic-planar lens. 
TABLE II

BME coefficients with $W / a=0.517$ normalized to $B_{00 x}$

\begin{tabular}{lcr}
\hline Coefficient & \multicolumn{1}{l}{$B_{p 0 x}$} & \multicolumn{1}{c}{$B_{p 2 x}=A_{p 2 y}$} \\
\hline 0 & 1 & $-9.016 e-3$ \\
1 & $-6.736 e-12$ & $2.026 e-4$ \\
2 & -0.027 & $-1.171 e-3$ \\
3 & 0.019 & $1.082 e-4$ \\
4 & 0.017 & $-5.470 e-5$ \\
5 & $5.942 e-4$ & $-7.764 e-5$ \\
6 & $-7.948 e-3$ & $-3.208 e-5$ \\
7 & $-5.612 e-3$ & $1.015 e-5$ \\
8 & $4.201 e-4$ & $2.474 e-5$ \\
9 & $4.240 e-3$ & $1.771 e-5$ \\
\hline
\end{tabular}

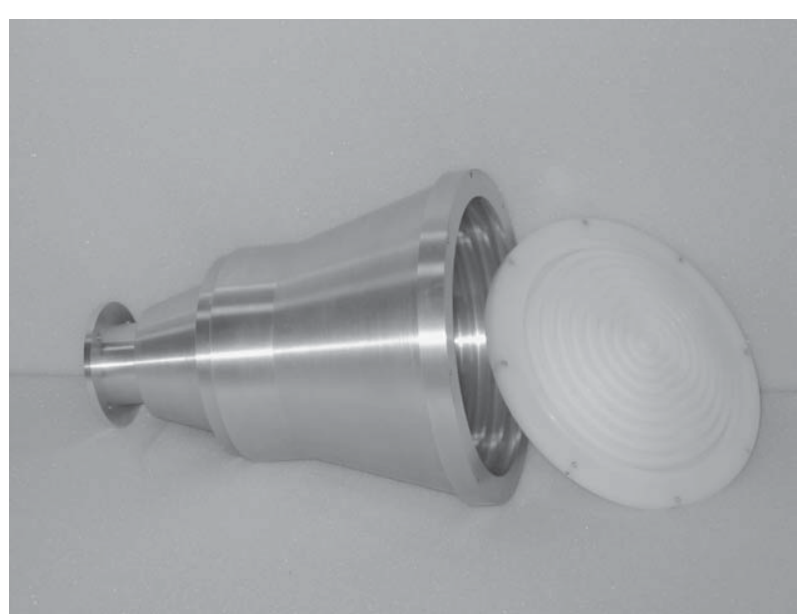

Figure 10. Photograph of the $7.25 \mathrm{GHz}$ corrugated conical horn and the hyperbolical-planar lens.

Beam patterns were measured for a corrugated conical horn working at a frequency of $7.25 \mathrm{GHz}$. The horn has a diameter of $157.0 \mathrm{~mm}$, an axial length of 261.66 $\mathrm{mm}$ and a half-flare angle of $16.7^{\circ}$ (Figure 9). The lens material is polypropylene with $n=1.5$. Radiation patterns were computed with GO and with QO using the fundamental mode. These are shown in Figure 11.

Figure 12 shows the optimum $W / a$ values for several horn geometries (half flare angle) and three lens materials.

The optimum $W / a$ value decreases with the half flare angle of the horn. In the limit where the horn has a half flare angle of zero, it does not need a phase correcting lens and the optimum $W / a$ result converges toward the BME proposed by Murphy for an isolated corrugated conical horn. 


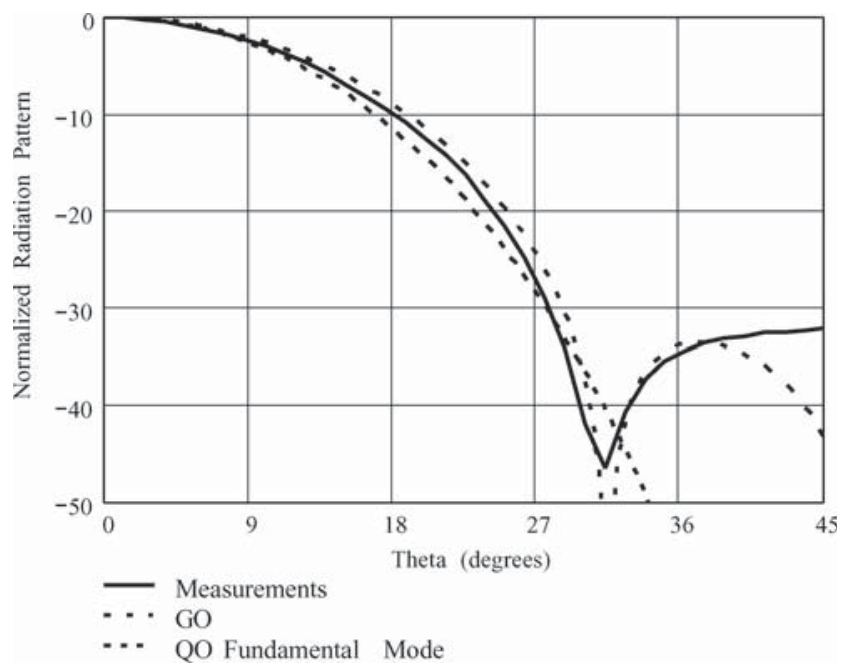

Figure 11. The 7.25 GHz (E Plane) measurements, GO and QO radiation patterns.

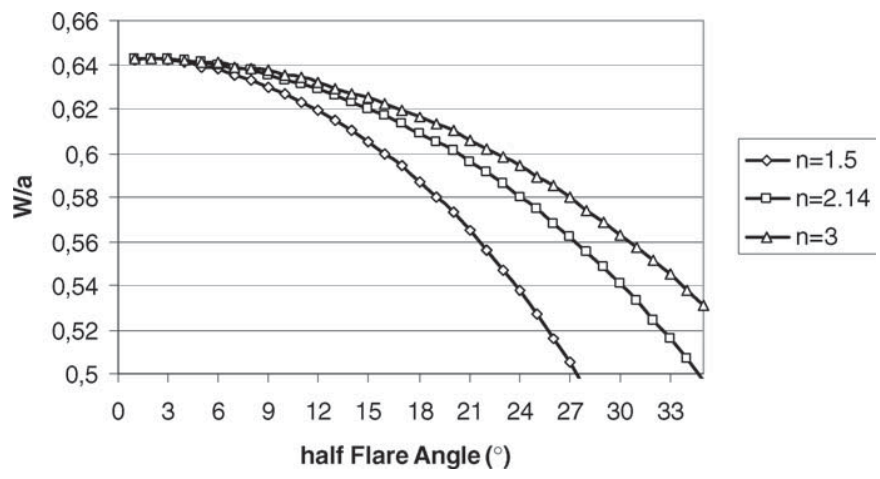

Figure 12. Optimum $W / a$ as a function of the half flare angle with three different materials for the hyperbolical-planar lens.

In the same way, the power in the fundamental mode for the optimum value of $W / a$ is shown in Figure 13. It shows the same convergent behavior as mentioned above. A saturation of the power carried by the fundamental mode with the half flare angle of the horn can be observed.

\section{Validation of the spherical-elliptical lens model: Application to the focalization of the new $40-\mathrm{m}$ radiotelescope of "Centro Astronómico de Yebes"}

To validate the usefulness of the QO-BME method, a receiver system is designed at three frequency bands for the new 40-m radiotelescope at the "Centro Astronómico 


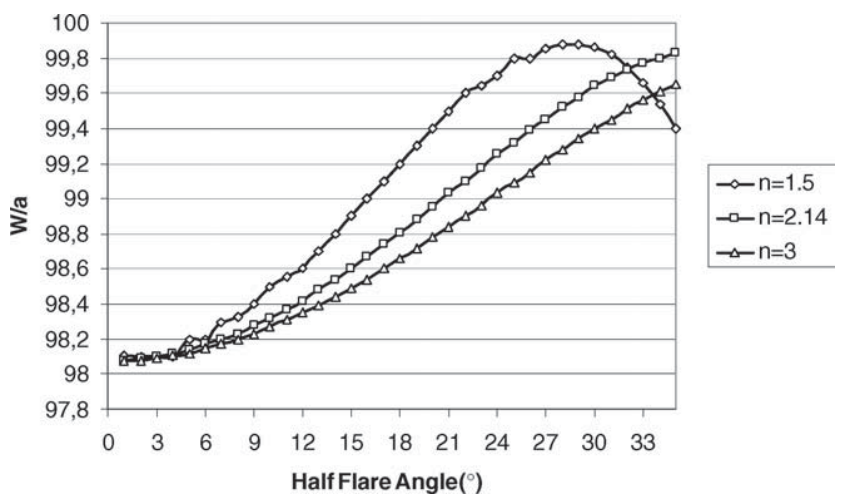

Figure 13. Optimum $W / a$ fundamental-mode power as a function of horn half-flare angle for three different hyperbolical-planar lens materials.

de Yebes". The optics proposed in this paper consists of a corrugated conical horn with a phase correcting lens placed in its mouth followed by an elliptical mirror. A spherical-elliptical lens is used.

The parameters used in the receiver design are defined in Figure 14. A classical Gaussian telescope (Goldsmith, 1997) design was developed, but in this case $d_{1}=0$, because the lens is placed in the mouth of the horn and corrects the horn phase error.

The radiotelescope has a main parabola diameter of $40 \mathrm{~m}$, an F/D equivalent of 7.9 and a subreflector diameter of $3.28 \mathrm{~m}$ (García et al., 2003). Three frequency bands, centred at: 22,30 and $45 \mathrm{GHz}$, have been studied. The material used for the lenses has a refractive index $n$ of 1.45 (Teflon).

A wavelength-independent system is achieved in all observation bands by means of the Gaussian beam telescope system (Goldsmith, 1997; Lamb, 1986).

The optimized optics for each of the three bands is summarized in Table III.

The edge taper and defocusing at the subreflector are found to be plane and frequency independent (Padman et al., 1987) for the three observation bands. Each one performs well. Our goal of a 12-dB edge taper is achieved over each design frequency band. Moreover, the subreflector defocus is nearly negligible for the

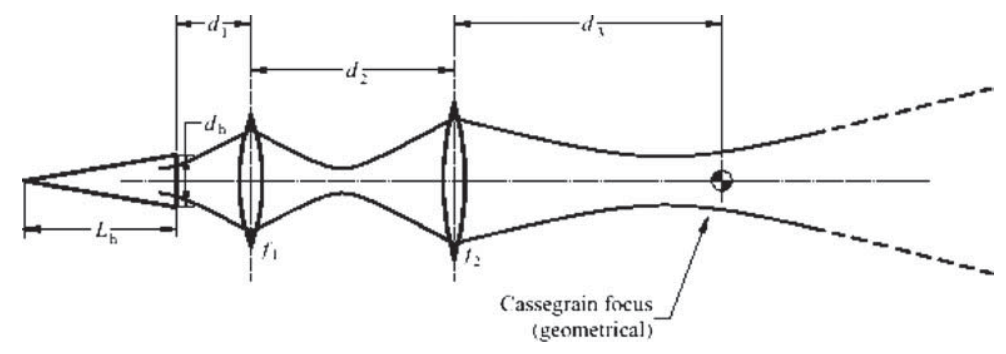

Figure 14. Geometry of the receiver optics. 
TABLE III

Optics for the channels with a hyperbolic lens in the mouth of the horn

\begin{tabular}{llll}
\hline & \multicolumn{3}{c}{ Frequency bands } \\
\cline { 2 - 4 } Parameters & $19-22-25 \mathrm{GHz}$ & $27-30-33 \mathrm{GHz}$ & $42-45-48 \mathrm{GHz}$ \\
\hline$d 2(\mathrm{~mm})$ & 498.1 & 665.9 & 397.8 \\
$d 3(\mathrm{~mm})$ & 488.7 & 649.3 & 391.7 \\
$f 1(\mathrm{~mm})$ & 131.8 & 140.4 & 134.8 \\
$f 2(\mathrm{~mm})$ & 488.7 & 649.3 & 391.7 \\
Axial length $(\mathrm{mm})$ & 111.0 & 105.3 & 70.0 \\
Horn radius $(\mathrm{mm})$ & 38.4 & 49.1 & 30.0 \\
\hline
\end{tabular}

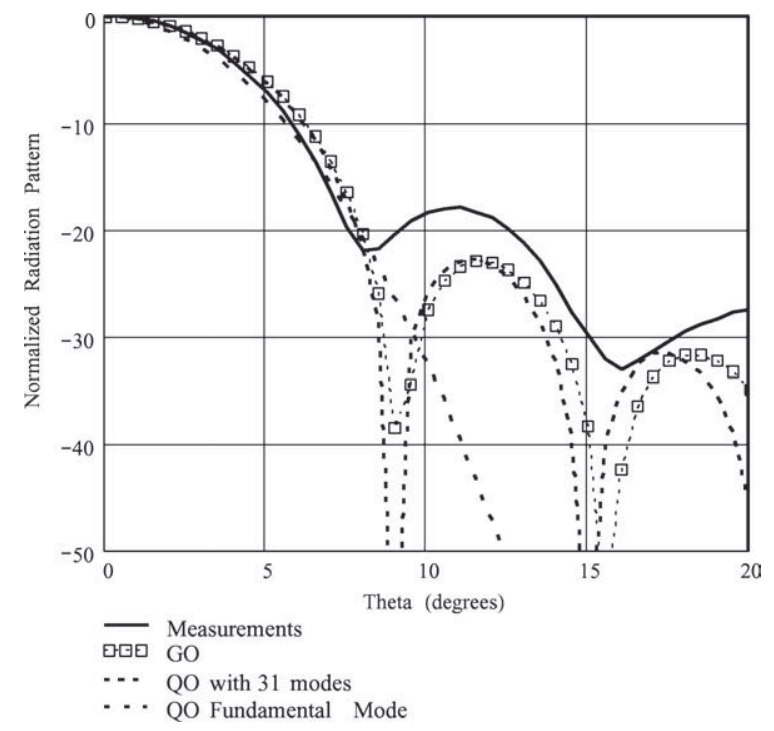

Figure 15. The $30 \mathrm{GHz}$ (E plane) measurements, GO and QO radiation patterns with the new BME are presented.

three observation bands (the radius of curvature is confined to within $\pm 0.1 \lambda$ for the three bands). The aperture efficiency is 0.8 and exhibits plane behavior in the bands implement (García et al., 2002).

To validate the model, a $30 \mathrm{GHz}$ radioastronomical receiver was built and analyzed using Equations (18) and (19).

Figure 15 shows the results of the QO theoretical model developed in this article, the theoretical pattern predicted by GO and measurements of a horn with a spherical-elliptical lens at $30 \mathrm{GHz}$ (Figure 16). The horn has an axial length of 


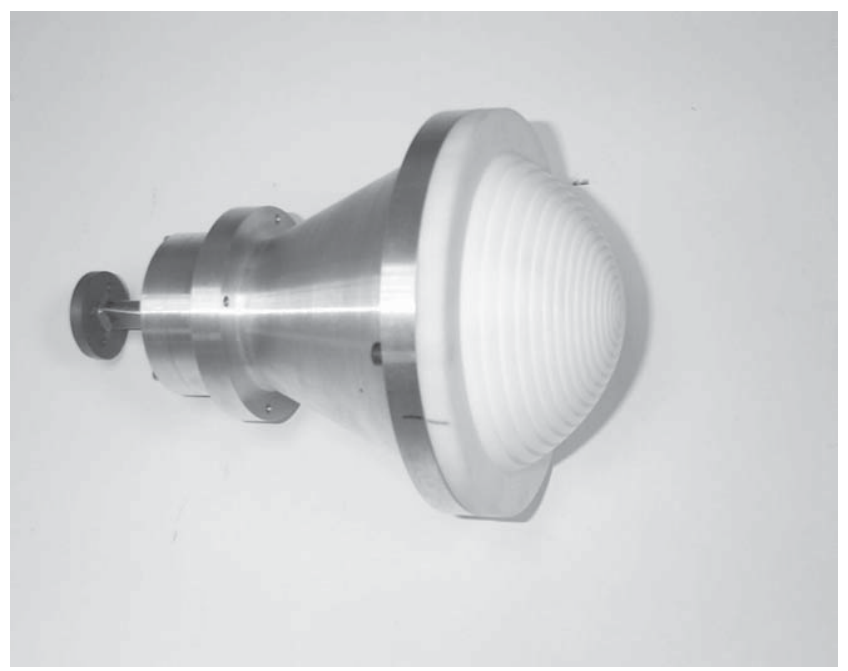

Figure 16. Photograph of the $30 \mathrm{GHz}$ corrugated conical horn and the spherical-elliptical lens.

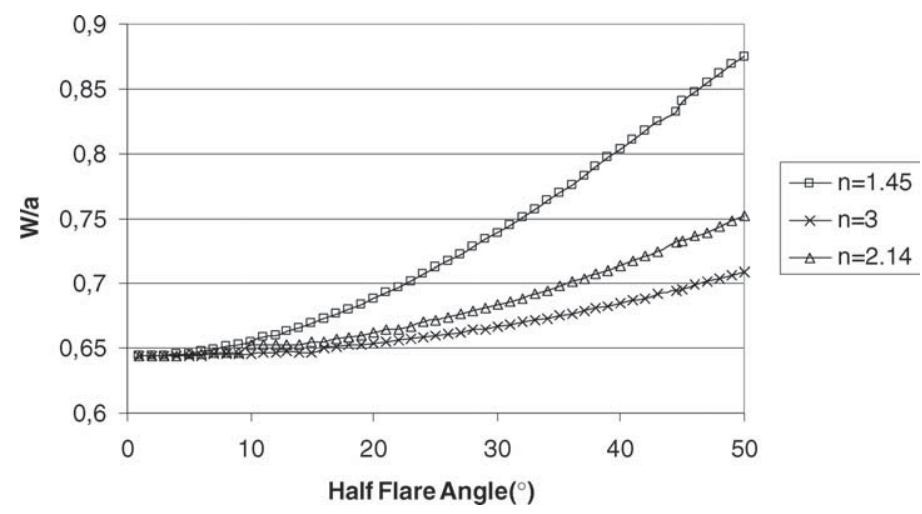

Figure 17. Optimum $W / a$ as a function of horn half-flare angle with three different materials for the spherical-elliptical lens.

$105.3 \mathrm{~mm}$, an aperture radius of $49.1 \mathrm{~mm}$ and the Teflon lens has a refractive index $n=1.45$.

It can be seen that the measured main beam is successfully modeled by QO. An important remark should be made. Apart from the effects considered in our model, the lens produces an additional attenuation due to reflections at lens surfaces and due to propagation within the lens material itself. Neither effect has been taken into account here since we get good matching by using corrugations and a low-loss lens material (Teflon). However, the matching is not perfect and produces higher sidelobe levels. These effects can be seen in measurements of Figure 15. 


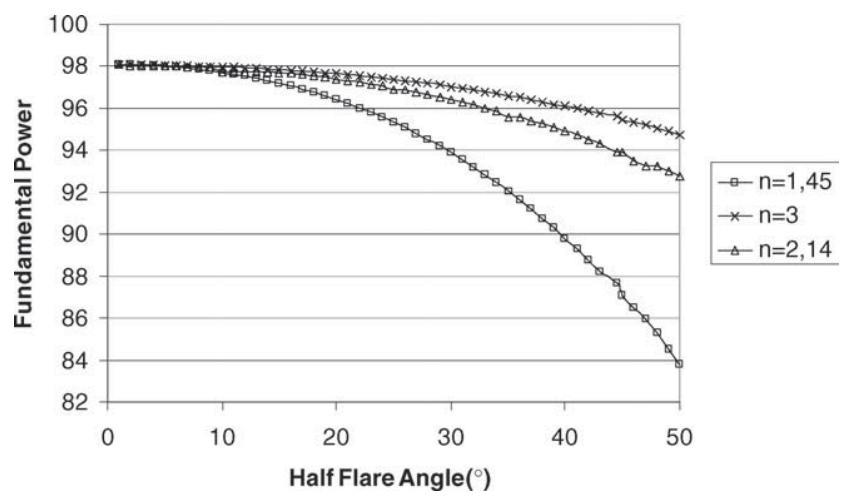

Figure 18. Optimum $W / a$ fundamental-mode power as a function of horn half-flare angle for three different spherical-elliptical lens materials.

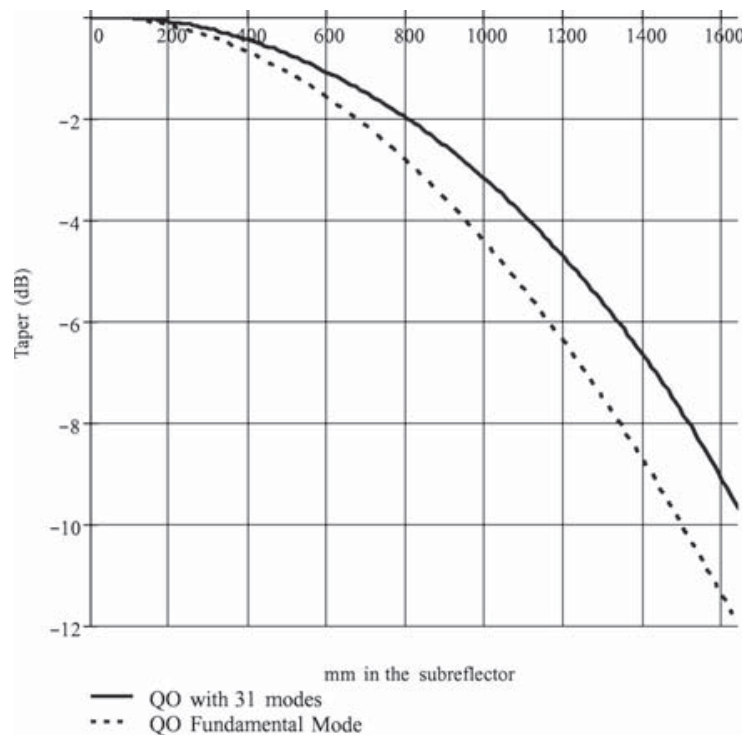

Figure 19. Subreflector illumination predicted by the fundamental mode model and 31-mode BME model.

Figure 17 shows the optimum $W / a$ values for several horn geometries (half flare angle) and three spherical-elliptic lens materials. The optimum $W / a$ value increases with the half flare angle. In the limit where a horn has a half flare angle of zero, it does not need a phase correcting lens, and the results converge toward the BME proposed by Murphy for an isolated corrugated conical horn.

In the same way, the power of the fundamental mode for the previous values of $W / a$ are shown in Figure 18. They exhibit the same convergent behavior as described previously. 


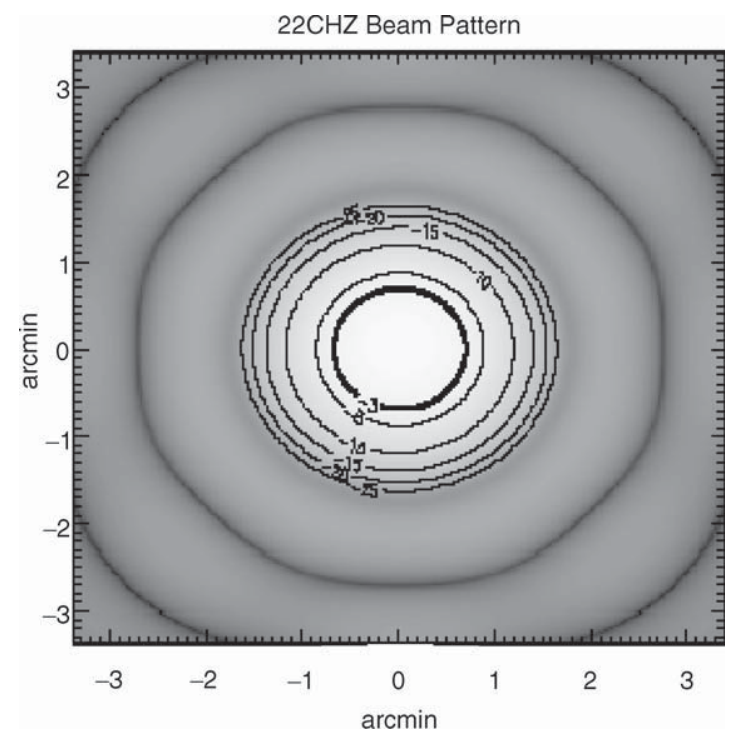

Figure 20. Copolar radiation pattern of the $22 \mathrm{GHz}$ channel. This was modeled using the paraxial software package GLAD.

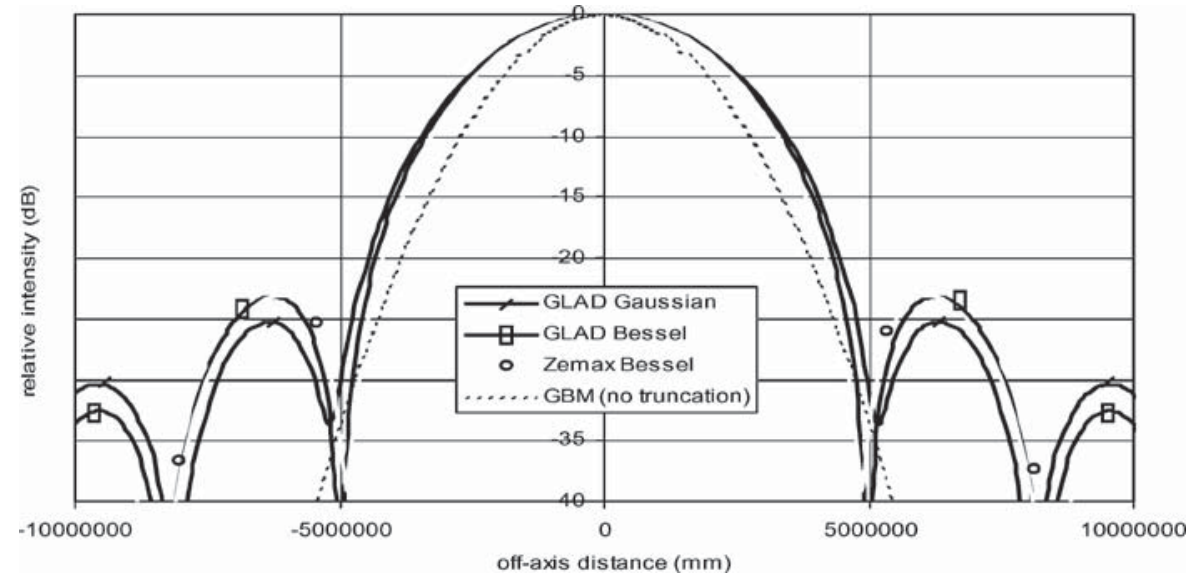

Figure 21. Copolar radiation pattern of the $22 \mathrm{GHz}$ channel simulated with GLAD and ZEMAX. A Gaussian and a Bessel function were used to model the output field of the horn. These are compared with the results of a simple fundamental Gaussian beam mode (GBM) model with no truncation at the optical components.

The illumination of the 40-m radiotelescope subreflector is shown in Figure 19. The subreflector is an hyperboloid of diameter $3.28 \mathrm{~m}$. A model considering only the fundamental mode predicts an edge taper of $-12 \mathrm{~dB}$, while if the same system is analyzed by means of our proposed BME with 31 modes, one observes that the taper is in fact $-10 \mathrm{~dB}$. 


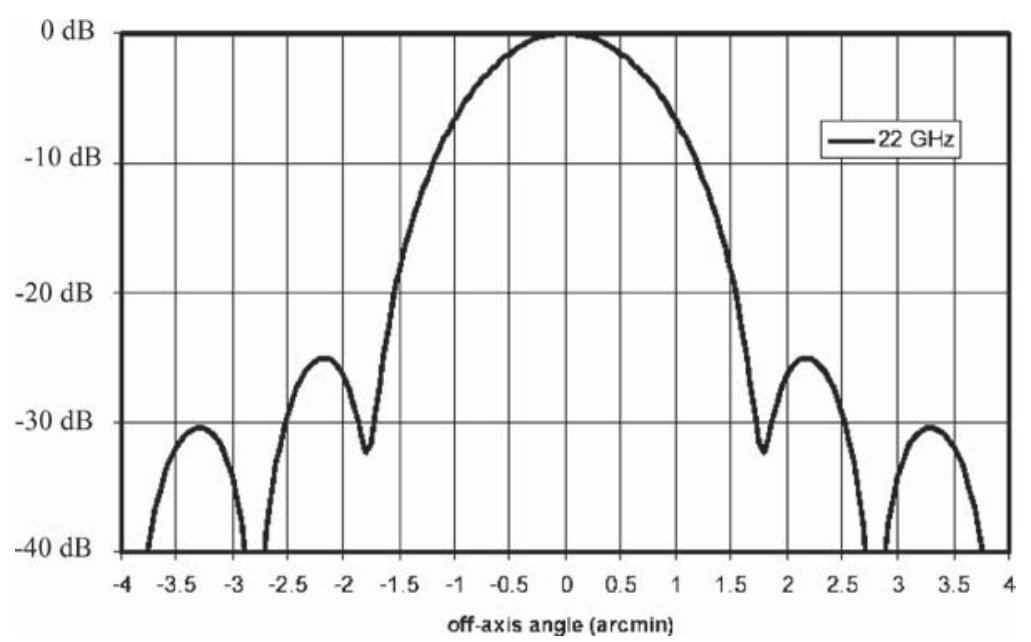

Figure 22. Copolar pattern radiation of the $22 \mathrm{GHz}$ channel predicted by GLAD.

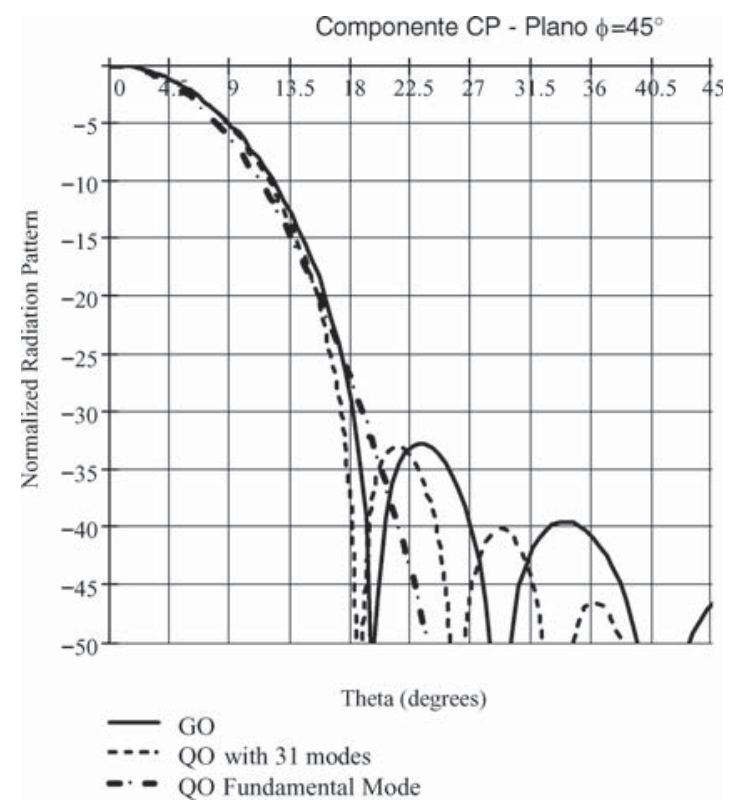

Figure 23. Simulated radiation pattern using the BME proposed (Copolar $45^{\circ}$ Plane).

However in our case this difference does not pose any problem. Typical optics and geometries, such as the ones presented in Section V, have an optimum edge illumination somewhere between -12 and $-10 \mathrm{~dB}$. For our designs an efficiency of 0.81 is obtained for the three observation bands by illuminating the edge at $-10 \mathrm{~dB}$. 


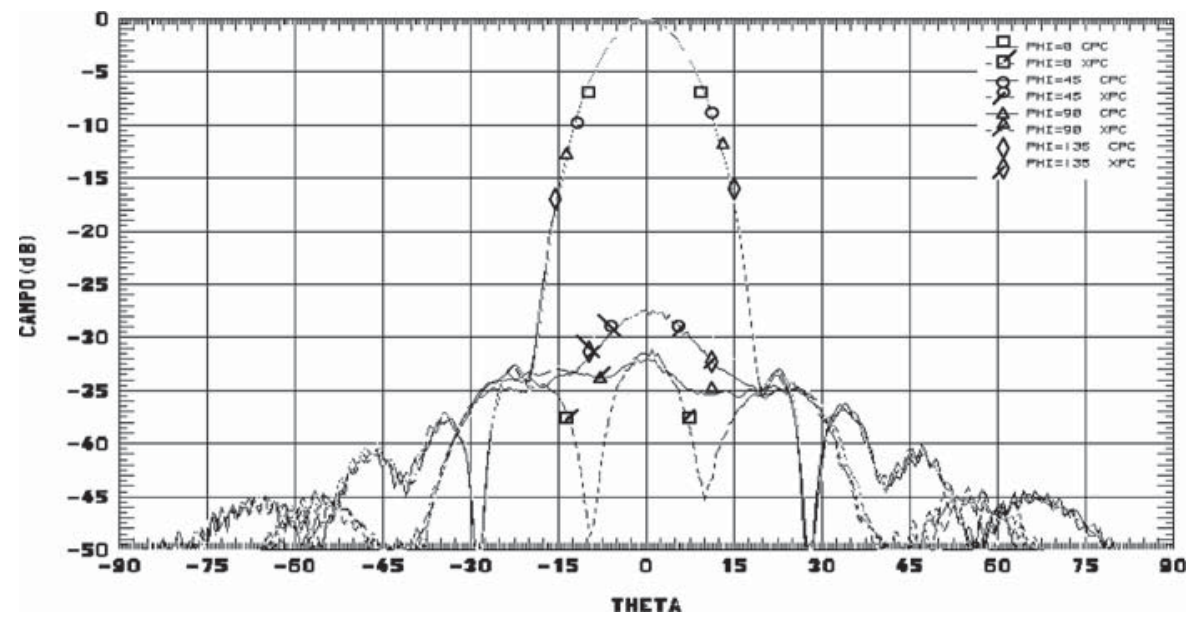

Figure 24. Measured radiation pattern of the horn and lens system with polarizer.

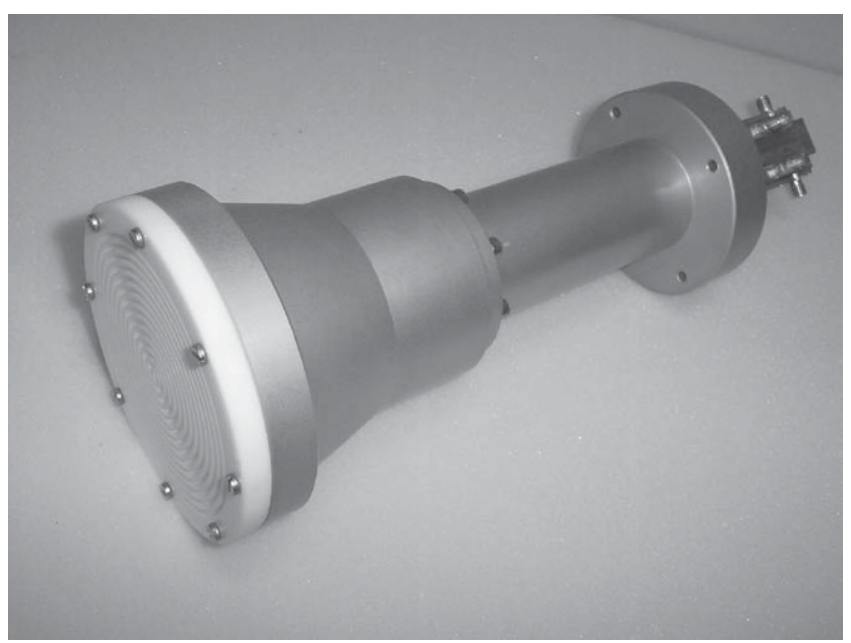

Figure 25. Photograph of the $22 \mathrm{GHz}$ horn and lens system.

\section{Implications for the design and measurement of the 22-GHz channel}

We have also constructed a horn and hyperbolical-planar lens for the 22-GHz channel. The 2D copolar radiation pattern for this channel of the telescope is shown in Figure 20. The simulation was carried out using the optical software package GLAD. In Figures 21 and 22 we present cuts of the copular far-field radiation pattern predicted by both GLAD and ZEMAX. The maximum of the cross-polar component is typically more than $25 \mathrm{~dB}$ below the on-axis copolar intensity. This is an important consideration when deciding whether to use the hyperbolic or 
spherical lens. The hyperbolic lens produces a lower crosspolar level. Also with a hyperbolic lens there are lower reflections in the material and this results in a better radiation patterns. Figure 25 shows a photograph of the $22-\mathrm{GHz}$ horn and the lens.

\section{Conclusions}

Placing the lens of a classical Gaussian telescope directly at the horn aperture has some mechanical advantages. The typical size of the horn plus lens system can be reduced and the lens-carrying holder within the cryostat can be simplified.

In the case of such near-field illumination of the lens, the model proposed by Wylde (1984) and Murphy (1988) should be improved and a new BME of the hornplus-lens is needed in order to get a better fit, comparable to, for instance, the GO solution.

This paper has developed a QO model for a corrugated conical horn with a phasecorrecting lens. The lens profiles considered in this paper are spherical-elliptical and hyperbolical-planar. The QO results have been compared with GO results and an agreement down to $-25 \mathrm{~dB}$ is achieved.

In all the focused bands and the validation models implemented in this paper, we have used the efficiency concept defined by Goldsmith (1997) and Wylde (1984). In all the radiotelescope bands, is achieved an independent wavelength system, because of the geometry used (Gaussian telescope; see Goldsmith, 1997). So, a $-12 \mathrm{~dB}$ taper edge in the subreflector is achieved in all the bands, and a wavelength independent focusing also is achieved. This $-12 \mathrm{~dB}$ value is a design objective in order to achieve an efficiency (see Wylde, 1984) of 0.81 wavelength independent.

It is important to remark that the configuration with pahse correcting lens could be optimal in a lot of cases of focusing radiotelescopes receivers. This is because of the stability in the tolerance position of the elements configuration (placement of the horn and the lens, errors in those placements). Also this kind of configuration is very stable in its electromagnetic properties when errors such as little changes in focal distance of lens occurs.

A comparison between the BME and GO models is presented by means of different radiation patterns for both types of lenses considered in this paper. Our model has been applied to the design of receivers at three observation bands: 22, 30 and $45 \mathrm{GHz}$. The receivers are those needed for the new 40-m radiotelescope in "Centro Astronómico de Yebes". Validation of the model has been achieved with the design and building of a corrugated conical horn and a hyperbolicalplanar lens at $7.25 \mathrm{GHz}$, described in Section 4, and at $22 \mathrm{GHz}$, described in Section 6. The spherical-elliptical lens model is validated using the telescope's $30-\mathrm{GHz}$ observation band receiver. 


\section{References}

Chu, T.-S.: July 1983, 'An imaging beam waveguide feed', IEEE Transactions on Antennas and Propagation, Vol. 31, No. 4, pp. 614-619.

Clarricoats, P. J. B. and Olver, A. D.: 1984, Corrugated horns for Microwave Antennas, Peter Peregrinus Ltd., chapters 2 and 4.

Clarricoats, P. J. B. and Saha, P. K.: 1969, Electron. Lett. 6(23), 592-593.

García, E., de Haro, L., López Fernández, J. A., Tercero, F., Galocha, B., Barcia, A. and Besada, J. L.: March 2002, Microwave Opt. Technol. Lett. 32(10), 420-423.

García, E., de Haro, L., López Fernández, J. A., Tercero, F., Galocha, B., Barcia, A. and Besada, J. L.: Junio 2003, 'Beam mode expansion applied to horn system with meniscus lens: New application to radioastronomy receivers', 2003 IEEE AP-S Antennas and Propagation/URSI Conference International Symposium, Columbus, Ohio, USA, Vol. I, pp. 136-139.

García, E., de Haro, L., López Fernández, J. A., Tercero, F., Galocha, B., Barcia, A. and Besada, J. L.: April 2003, Microwave Opt. Technol. Lett., 121-123.

García, E., de Haro, L., López Fernández, J. A., Tercero, F., Galocha, B., Barcia, A. and Besada, J. L.: August 2002, Int. J. Infrared Millimetre Waves 23(8), 1193-1213.

Goldsmith, P. F.: 1997, Quasioptical Systems, IEEE Press, chapters 2-4.

Jasik, H., 1961, Antenna Engineering Handbook, Mc Graw-Hill, chapter 14.

Kildal, P. S., Jakobsen, K. and Subhakar, R. K.: 1984, 'Meniscus les corrected corrucated horn: A compact feed for a Cassegrain antenna,' IEE Proceedings, Vol. 131, Pt. H, no. 6, pp. 390-394.

Lamb, James W.: April 1986, Int. J. Infrared Millimeter Waves 7(10), 1511-1536.

Milligan, Thomas A.: 1985, Modern Antenna Design, McGraw Hill Book Company, chapters 2 and 3.

Murphy, J. A.: April 1988, 'Aperture efficiencies of large axisymmetric reflector antennas fed by conical horns', IEEE Transactions on Antennas and Propagation, Vol. 36, No. 4, pp. 570-575.

Padman, R., Murphy, J. A. and Hills, R.: October 1987, 'Gaussian mode analysis of cassegrain antennas efficiency', IEEE Transactions on Antennas and Propagation, Vol. 33, Issue 10, pp. 1093-1103.

Tuovinen, J., Hirvonen, T. M. and Raisanen, A. V.: June 1992, 'Near-field analysis of a thick lens and horn combination: Theory and measurements', IEEE Transactions on Antennas and Propagation, Vol. 40, No. 6, pp. 613-619.

Wylde, R. J.: August 1984, 'Millimetre wave Gaussian beam-mode optics and corrugated feed horns', IEE Proceedings, Vol. 131, Pt. H., No. 4, pp. 258-262. 